\title{
Uso de recursos digitales en la formación de los ingenieros en el área de ciencias básicas
}

\section{Uso de recursos digitales en la formación de los ingenieros en el área de ciencias básicas}

DOI: $10.46932 / \mathrm{sfjdv} 2 \mathrm{n} 2-192$

Received in: March 1st, 2021

Accepted in: May 30th, 2021

\begin{abstract}
M T.E Lorena Mendoza Guzmán
Instituto Tecnológico Superior del Occidente Estado de Hidalgo.

Paseo del agrarismo 2000. Carr. Mixquiahuala-Tula, Km 2.5 Mixquiahuala de Juárez, Hidalgo, C.P. 42700. México

E-mail: lmendozag@itsoeh.edu.mx
\end{abstract}

\section{Mtro. En C. Juan Adolfo Álvarez Martínez}

Instituto Tecnológico Superior del Occidente Estado de Hidalgo.

Paseo del agrarismo 2000. Carr. Mixquiahuala-Tula, Km 2.5 Mixquiahuala de Juárez, Hidalgo, C.P. 42700. México

\section{M.T.I Cristy Elizabeth Aguilar Ojeda}

Instituto Tecnológico Superior del Occidente Estado de Hidalgo.

Paseo del agrarismo 2000. Carr. Mixquiahuala-Tula, Km 2.5 Mixquiahuala de Juárez, Hidalgo, C.P. 42700. México

\section{Mtro. Rolando Porras Muño}

Instituto Tecnológico Superior del Occidente Estado de Hidalgo.

Paseo del agrarismo 2000. Carr. Mixquiahuala-Tula, Km 2.5 Mixquiahuala de Juárez, Hidalgo, C.P. 42700. México

\section{Doc. En C y AD Jorge Luis Rodríguez Ruiz}

Instituto Tecnológico Superior del Occidente Estado de Hidalgo.

Paseo del agrarismo 2000. Carr. Mixquiahuala-Tula, Km 2.5 Mixquiahuala de Juárez, Hidalgo, C.P. 42700. México

\section{RESUMEN}

En la actualidad existe un creciente uso de la tecnología en la educación como apoyo al proceso de enseñanza-aprendizaje que permite desarrollar las competencias básicas, disciplinares y profesionales de los alumnos para obtener el mejoramiento de sus habilidades que potencialicen la consolidación del conocimiento. Por tal motivo se ha implementado el uso de las plataformas educativas en las aulas del Instituto Tecnológico Superior del Estado de Hidalgo.

El uso de las TI como apoyo dentro del aula es altamente favorable en el área de ciencias básicas debido a la aplicación de los recursos digitales, el empleo de plataformas educativas de acceso libre y plataformas educativas para la enseñanza de las matemáticas; estos recursos permiten tanto la mejora de la comprensión de los temas para los diversos estilos de aprendizaje como la innovación de la práctica docente; la elección de los materiales requiere del conocimiento tanto de aspectos pedagógicos, disciplinares, de aplicaciones móviles, tecnologías emergentes, sistemas de gestión de aprendizaje, aprendizaje adaptativo y recursos educativos abiertos entre otros; además de seleccionar el material, el 
docente debe de implementar las TI en una secuencia o plan de trabajo con la finalidad de administrar adecuadamente el uso de estos recursos teniendo en cuenta las ventajas y desventajas de los diferentes recursos digitales, de tal manera que permita mejorar el desempeño académico de los estudiantes en el área de ciencias básicas.

El uso de la tecnología tiene en cuenta como elemento principal al estudiante; este proceso se denomina aprendizaje centrado en el alumno, que se refiere al modelo de aprendizaje donde el estudiante debe desarrollar sus capacidades y aplicarlas en situaciones que el profesor le deba plantear.

Para ello el profesor debe tener el conocimiento si los estudiantes cuentan con dispositivos móviles, tabletas, computadoras personales o de escritorio con enlace a internet y cuáles son sus capacidades de almacenamiento o de reproducción de audio, video y texto; a partir del conocimiento adquirido, el docente elegirá y diseñará los recursos didácticos y los implementará en la plataforma de la institución educativa, esto conlleva a que el alumno cuente con el material en todo momento lo que le permitirá reforzar los conocimientos obtenidos en el aula dando paso al incremento de los índices de aprovechamiento.

\begin{abstract}
Currently there is a growing use of technology in education as a support to the teaching-learning process that allows the development of basic, disciplinary and professional competencies of students to obtain the improvement of their skills that potentiate the consolidation of knowledge. For this reason, the use of educational platforms has been implemented in the classrooms of the Instituto Tecnológico Superior del Estado de Hidalgo.

The use of IT as a support in the classroom is highly favorable in the area of basic sciences due to the application of digital resources, the use of free access educational platforms and educational platforms for the teaching of mathematics; these resources allow both the improvement of the understanding of the topics for the different learning styles and the innovation of the teaching practice; the choice of materials requires knowledge of pedagogical and disciplinary aspects, mobile applications, emerging technologies, learning management systems, adaptive learning and open educational resources among others; in addition to selecting the material, the teacher must implement IT in a sequence or work plan in order to properly manage the use of these resources taking into account the advantages and disadvantages of the different digital resources, so as to improve the academic performance of students in the area of basic sciences.

The use of technology takes into account the student as the main element; this process is called studentcentered learning, which refers to the learning model where the student must develop their skills and apply them in situations that the teacher should raise.

For this, the teacher must have the knowledge if the students have mobile devices, tablets, personal or desktop computers with internet link and what are their storage capacities or audio, video and text reproduction; from the knowledge acquired, the teacher will choose and design the didactic resources and implement them on the platform of the educational institution, this leads to the student having the material at all times which will allow him to reinforce the knowledge obtained in the classroom giving way to the increase of the rates of achievement.
\end{abstract}

\title{
1 INTRODUCCIÓN
}

Es muy conocido que los resultados de desempeño académico de los estudiantes de ingeniería en el área de matemáticas y en ciencias básicas en el nivel superior son poco favorables según muestran los indicadores estadísticos que publican las instituciones en sus registros de eficiencia terminal. Dichos 
resultados son atribuibles a una diversidad de factores que van desde los socioemocionales hasta los pedagógicos y disciplinares.

También es ampliamente sabido que se han hecho múltiples esfuerzos para revertir esta situación, y para ello desde diferentes ejes de trabajo se han implementado estrategias que van desde el cambio de modelos educativos, capacitación en estrategias de aprendizaje, dotación de infraestructura, uso de recursos didácticos digitales, hasta cambio de las teorías de aprendizaje.

No está por demás exaltar la importancia que el aprendizaje de las ciencias como herramienta le permitirá al futuro ingeniero afrontar, decidir, resolver y proponer soluciones a situaciones en las que se requiere de dicho conocimiento, mas sin embargo en los primeros niveles de licenciatura el estudiante novel en éstas áreas no lo percibe así, por lo que también en el proceso de enseñanza y aprendizaje se tiene que luchar en contra de éstas ideologías que en algunos casos están fuertemente arraigadas en las comunidades estudiantiles.

Ahora bien, para el caso específico de la enseñanza de la matemática como campo de estudio encontramos diferentes enfoques que nos permiten entender el proceso de aprender matemáticas; existiendo líneas de investigación que van desde la epistemología, la heurística, la didáctica, los procesos cognitivos, hasta el uso de la tecnología; todas ellas enfocadas a conocer y mejorar el proceso educativo en la formación de los ingenieros en el área de ciencias básicas.

En particular este trabajo aborda el eje temático del uso de la tecnología para la enseñanza y aprendizaje de la matemática; su campo de estudio surge por el mismo hecho de que en esta sociedad del conocimiento los alumnos tienen a su alcance una diversidad de dispositivos tecnológicos los cuales abren la alternativa de apoyar el proceso de aprendizaje.

El punto medular es cómo y cuándo integrar toda esta amplia cantidad de medios a favor de una mejora del proceso.

Ante todo, es importante mencionar que detrás del uso de los dispositivos como la computadora, la tableta, el teléfono, el internet, las redes sociales o la televisión por cable debe existir el conocimiento fundamentado que valida su uso en el aspecto escolar, esto permitirá dirigir los esfuerzos del profesorado y de las instituciones por una senda que ayude a incrementar los índices de aprovechamiento.

\section{TEORÍA}

A lo largo de la historia han existido diversos enfoques y teorías que fundamentan el uso de recursos didácticos para aprender conceptos y procedimientos en diversas ramas de la ciencia; desde los objetos físicos manipulables, hasta los que hoy en día son recursos digitales que simulan, grafican y en los que se pueden manipular variables en el estudio de fenómenos complejos. 
La cantidad de materiales digitales existentes que son accesibles para usar en la educación son muy variados; casi cualquier persona con acceso a una computadora e internet puede obtener conocimientos y educarse por sí misma.

Sin embargo, habremos de decir en principio que el proceso de enseñanza y aprendizaje para poder brindar resultados satisfactorios requiere del análisis y conocimiento de tres elementos inseparables: el educando o estudiante; el profesor o docente y el conocimiento. De la importancia de estos elementos se hablará en la siguiente sección.

En cuanto al primer punto de los componentes que integran el proceso (el estudiante) es importante mencionar que para que haya una implementación eficaz de la tecnología se hace necesario enfatizar el hecho de que dicha tecnología a implementar debe focalizarse hacia el alumno, es decir sea el centro de atención y ello implica que no únicamente le permita ser un espectador o lector de los materiales, sino que le posibilite interactuar, modificar, participar, e incluso evaluarse a través de ellos.

Este tipo de modelo de aprendizaje del uso de la tecnología teniendo en cuenta como elemento primordial al estudiante se denomina aprendizaje centrado en el alumno, que se refiere al esquema de trabajo donde el estudiante desarrolla sus capacidades y las aplica en situaciones que el profesor le plantea.

Así surge la ya tan conocida educación basada en competencias, donde el conocimiento mismo no es suficiente como teoría sino la importancia radica en la aplicabilidad o usabilidad de dicho conocimiento para resolver problemas que requieren de observación, análisis, decisión, argumentación, todo ello en un contexto integral que permita valorar el desempeño del alumno.

En relación al segundo elemento (el profesor) se puede citar los modelos donde el aprendizaje está basado en la enseñanza, el cual debería haber quedado atrás ya hace muchos años; en este modelo el profesor era el experto y el que dominaba todo el conocimiento que el alumno debía aprender; sin embargo, esta forma de trabajo es una actividad sumamente arraigada y que aún se encuentra muy recurrida por el profesorado.

De la misma manera el aprendizaje centrado en los medios también es un modelo que ya se encuentra en desuso, y donde se suponía que la calidad del material de texto o recurso didáctico dictaminaba el éxito del aprendizaje. En estos casos los modelos estaban basados en la importancia que se les daba a los recursos de aprendizaje.

Un punto a considerar que es de suma relevancia que se refiere al alumnado es el tipo de estilos de aprendizaje; aquí hay que tomar en cuenta que no todos los estudiantes aprenden de la misma manera, y para ello los materiales a elegir deben tener en cuenta a los alumnos cuyo canal de recepción de información es visual, en este caso se prioriza el uso de recursos como el video, las infografías, organizadores gráficos y material en formato de imágenes ya sea en modo estático o gráficos dinámicos. 
Otro de los canales de comunicación es el auditivo, para lo cual deben considerarse materiales que incluyan información en forma de audio como los podcast.

Para concluir este punto se deben también diseñar y emplear materiales para alumnos kinestésicos es decir los que requieren de trabajar mediante la manipulación de objetos, es decir aprenden haciendo y trabajando con los materiales, para ello son de gran apoyo el software para graficación, animación y simulación de procesos.

Otro de los factores a tener en cuenta es el tipo de soporte tecnológico que se va a usar para que el alumno trabaje con dichos recursos digitales, estos recursos son mejor conocidos como OIA (objetos interactivos de aprendizaje), para ello también ha de tenerse la información sobre si se hace necesario emplear algún sistema de gestión de aprendizaje (LMS) o algún entorno virtual de aprendizaje (EVA), esto es algún sistema (plataforma educativa) donde se encuentren alojados los OIA.

En este sentido es que aparece como elemento central la decisión de usar o no las denominadas plataformas educativas, en las cuales se integran los materiales a los que los alumnos tendrán acceso.

Para ello debe tenerse el conocimiento si los estudiantes cuentan con dispositivos móviles y cuáles son sus capacidades de almacenamiento o de reproducción de audio, video y texto; Dentro de dichos dispositivos a considerar están los teléfonos, las tabletas o las computadoras con enlace a internet.

En la actualidad existe una diversidad de plataformas educativas tanto gratuitas como comerciales con características para realizar las siguientes funciones: alojamiento de OIA, creación de cuestionarios y exámenes, generación de foros de discusión, seguimiento de entrega de trabajos en la misma plataforma, control de calificaciones, comunicación síncrona o asíncrona.

La segunda opción que implica el uso de los OIA es que el profesor diseñe o integre el material en un sistema de navegación móvil donde el material pueda ser consultado de manera asíncrona por el estudiante, es decir tenga un dispositivo de almacenamiento portátil para accesar a la información.

Actualmente muchas plataformas tienen versiones móviles para asegurar el flujo de la información en todo momento.

Para este caso donde el material de estudio sea portable, se denomina m-mobile (aprendizaje móvil) donde el recurso o medio es un dispositivo como teléfono o tableta sin necesidad de que los recursos se encuentren alojados en una plataforma educativa.

La ventaja de poder emplear en la medida de lo posible una plataforma educativa como sistema de gestión de aprendizaje es que en ella pueden integrarse grupos de discusión acerca de un tema que permita a los alumnos participar, argumentar o debatir, lo que es un aspecto esencial en su desarrollo; También permite dejar evidencia a manera de historial de la participación de los alumnos en las actividades logrando con ello un mejor control y optimización del tiempo y recursos. 
Otro beneficio de usar un sistema LMS es que el profesor puede programar sus actividades y evaluaciones para fechas específicas, aperturandolas en el momento indicado de acuerdo a su secuencia didáctica; pueden citarse en dos tipos las ventajas del uso de los sistemas LMS, en el aspecto académico para la retroalimentación, comunicación y cátedra; y en el aspecto administrativo para el control y seguimiento de las evaluaciones de los estudiantes.

De esta forma es que se han generado las variantes de aprendizaje basado en las TIC que se pueden clasificar como b-learning (blended learning) aprendizaje mixto, que se refiere a actividades en aula y que se complementan por otras tareas en plataforma o en un LMS; el e-learning (cursos totalmente en línea, es decir educación no presencial) o el ya mencionado m-mobile.

En cualquiera de los tres casos se requiere que el profesor establezca de antemano la secuencia de las actividades, las instrucciones y fechas en que el alumno deberá consultar o trabajar en determinados materiales; esto es lo que se denomina diseño instruccional; que se refiere a la forma, cantidad, etapas y programación de las actividades del curso. En esta parte se requiere al menos el conocimiento básico de los tipos de modelos de diseño instruccional entre los cuales podemos mencionar el modelo ASSURE o el modelo ADDIE.

El diseño instruccional es importante en el proceso dado que en la propuesta de instrucción se fundamenta el uso de los distintos elementos y recursos didácticos que deben ser cuidadosamente identificados, diseñados y desarrollados de forma que faciliten y apoyen la construcción de conocimientos y habilidades del alumno.

En general podemos mencionar que un modelo de diseño instruccional debe considerar los siguientes elementos:

- Análisis de los estudiantes:

- Establecimiento de los objetivos

- Selección de métodos, medios y materiales

- Forma de utilización de los medios y materiales

En cuanto al factor o punto para definir la cantidad y calidad de los materiales a usar, es importante decidir si los recursos han de ser de autoría propia o bien obtenidos de repositorios digitales que pueden ser los denominados REA (recursos educativos abiertos), en este último caso hay que considerar si dichos materiales son de uso público y pueden ser accesados únicamente en línea o pueden ser almacenados en dispositivos de almacenamiento portátil.

La desventaja del empleo de los REA es que, en determinadas circunstancias, los servidores donde se encuentra la información están bajo control externo (fuera del sistema LMS), por ello el profesor debe 
verificar de manera constante la existencia de la información y sobre todo que esté vigente para el momento en que va a ser utilizada.

Otro de los puntos a considerar para la implementación de las TI es el seguimiento y valoración del aprendizaje, para lo cual existe software para el diseño de cuestionarios interactivos que permiten reafirmar los conocimientos; en este caso hay que verificar que el sistema LMS o medio de almacenamiento portátil donde se encuentra la información sea de fácil interacción y manejo.

Entre los aspectos esenciales a considerar para la implementación de las TI en el proceso de enseñanza y aprendizaje, esta la definición de los objetivos que se espera lograr, lo cual va desde un análisis de las razones por las que se ha de emplear las TI hasta el tipo de infraestructura con la que cuenta tanto en la institución como los mismos alumnos.

A continuación, se hace una descripción de los factores a considerar para la implantación de las TI para la enseñanza y el aprendizaje de las matemáticas y de las ciencias.

- Tipo de Modelo educativo: Centrado en el profesor, centrado en los materiales, centrado en el alumno.

- Tipo de modelo de diseño instruccional: ASSURE, ADDIE.

- $\quad$ Tipo de estilos de aprendizaje: visual, auditivo, kinestésico.

- Tipo de sistema LMS para gestión de aprendizaje: Definir si se requiere el uso de plataformas educativas.

- Tipo de metodología de aprendizaje: e-learning, b-learning, m-learning

- Uso de materiales: De diseño propio o de uso público.

\section{PARTE EXPERIMENTAL}

De todos los elementos que se requieren para diseñar una propuesta de un curso a nivel de ingeniería basado en el uso de las TI, en el Instituto Tecnológico Superior del Occidente del Estado de Hidalgo, en particular fue importante y necesario hacer un análisis de la infraestructura existente, del conocimiento y de las capacidades que tenían los alumnos en el uso de tecnología, así como del acceso a una red de comunicación.

A través de un cuestionario aplicado a los estudiantes se obtuvo la información que permitió definir y tomar la mejor decisión respecto al uso de un sistema LMS y del tipo de recursos y materiales a utilizar.

Los resultados fueron los siguientes: 
- Mas del $80 \%$ de los estudiantes usan teléfono para obtener información de internet para complementar o investigar temas relativos a las asignaturas que cursan.

- Mas del $60 \%$ conoce y ha utilizado con anterioridad una plataforma educativa para realizar actividades de asignaturas que cursa.

- Mas del 95\% emplea recursos y software para realizar y presentar trabajos escolares.

- Mas del 70\% visualiza videos y tutoriales en plataformas, como medio de aprendizaje o investigación.

- Más del 60\% tiene acceso a una red de internet ya sea en casa o datos móviles.

- Entre el $30 \%$ y $60 \%$ usa el teléfono para descargar las tareas o enviarlas.

- La institución cuenta con red de internet en biblioteca y laboratorios de cómputo que están disponibles a los estudiantes.

- La institución, en particular la sección de Ingeniería en sistemas computacionales cuenta con un servidor para alojar un sistema LMS.

Con estos registros estadísticos se planteó que lo conveniente fue el Contar con un sistema LMS aprovechando la oportunidad de que el área académica cuenta con la tecnología de un servidor y red de acceso a datos para alojar una plataforma.

En cuanto a la forma de trabajo y considerando que es un sistema de educación presencial, la implementación de la tecnología se basó en el modelo centrado en el alumno bajo la metodología de elearning, es decir la combinación de elementos didácticos presenciales en aula y actividades en la plataforma educativa.

En relación al modelo de diseño, se usó como referencia los elementos propuestos en la metodología ADDIE que es en general el que proporciona los mejores beneficios, considerando que dada sus ventajas es usada ampliamente casi de manera generalizada como un estándar en el diseño de cursos en línea, no solamente en el contexto de educación formal sino también en el ámbito laboral para la capacitación de personal en las organizaciones y empresas.

De esta manera, la estructura de las asignaturas en las que se empleó la tecnología tuvo como contenido actividades para que el alumnado consultara la información en forma síncrona y asíncrona teniendo como referencia el temario de la asignatura.

La comunicación síncrona se llevaba a cabo mediante sesiones de videoconferencia empleando algunas de las plataformas disponibles en la red. 
Los materiales para abordar los temas se diseñaron considerando los diversos estilos de aprendizaje, de manera que se crearon algunos recursos de tipo multimedia, también se generaron recursos en formato textual y gráfico, empleando en algunos casos material de repositorios REA.

Para mejorar el aprendizaje se tuvo como apoyo el uso de software para simulación y animación en donde los alumnos pudieron practicar los conocimientos.

Estos recursos se emplearon como medio de reforzamiento y también como elementos de validación de resultados, lo cual permitió que los alumnos mejoraran su confianza al asegurarse que las soluciones de los problemas planteados eran correctas.

Los estudiantes estuvieron trabajando en sus sesiones de clase de acuerdo con el calendario escolar y las actividades en plataforma estuvieron disponibles para cuando los estudiantes las requerían.

En relación a la comunicación con los estudiantes, en plataforma se establecieron foros de discusión donde se planteaban las dudas y exponían los temas, de manera que los estudiantes participaban y obtenían la información actualizada para realizar las actividades.

Para la fase de evaluación, se estableció un conjunto de actividades en las que los estudiantes contestaban exámenes, tanto para retroalimentar sus conocimientos como para obtener una puntuación enfocada a su calificación del tema.

Dichas evaluaciones se diversificaron, se aplicaron en línea, mediante cuestionarios interactivos, realizando exposiciones de sus proyectos donde los explicaban y agregaban sus archivos en dicha plataforma.

\section{RESULTADOS}

La puesta en marcha de la prueba piloto requirió de los conocimientos de diversas áreas tanto tecnológicas como pedagógicas; una de las ventajas en la implementación es que los estudiantes ya tenían un conocimiento del uso de diversas tecnologías, sin embargo, el uso de diversos recursos a abierto la posibilidad de potencializar el proyecto para expandirlo a una cantidad mayor de asignaturas usando las TI teniendo en cuenta la experiencia de este trabajo.

Los resultados obtenidos al usar un sistema LMS para integrarlo como elemento en el proceso de enseñanza y aprendizaje en algunas de las asignaturas de ciencias básicas fueron los siguientes.

El porcentaje de alumnos aprobados en los cursos estuvo entre el $70 \%$ y $90 \%$. Respecto a este indicador se hizo un análisis detallado y se pudo observar que los estudiantes que no aprobaron los cursos fueron por causas ajenas como, por ejemplo, se dieron de baja por circunstancias fortuitas, factores económicos, falta de interés en la carrera, etc. 
El porcentaje de satisfacción en el uso de sistema LMS: osciló entre el 60\% y 75\%, lo que dio como pauta de interés el saber que los recursos usados fueron en general de la aceptación de los estudiantes y les permitió el logro de los aprendizajes marcados en el temario.

Las asignaturas en las que se empleó la plataforma de gestión de aprendizaje LMS fueron probabilidad y estadística, cálculo integral y matemáticas discretas que pertenecen a los primeros semestres de las carreras de ingeniería en sistemas computacionales en el Instituto.

Dentro de las oportunidades de análisis que se tuvieron en la implementación de la tecnología en la enseñanza y aprendizaje de las ciencias es que al ser estas asignaturas de tipo teórico-procedimental se pudo tener información sobre el nivel de aprendizaje obtenido en el contexto conceptual usando recursos digitales para posteriormente aplicarlo a la solución de casos prácticos.

\section{CONCLUSIONES}

Uno de los objetivos fundamentales del aprendizaje de las matemáticas y de las ciencias es lograr que el alumnado use y desarrolle un conjunto de conocimientos que les sean útiles en situaciones que se encuentran en contextos muy diversos, sin embargo, como la sociedad actual se encuentra en constante cambio, dichos conocimientos deben ser adaptativos y flexibles para permitir que los estudiantes adquieran las competencias y las empleen eficazmente.

El objetivo ancestro de que el aprendizaje de los contenidos fuera el último fin, ha quedado obsoleto si no se encuentra que dicho conocimiento tenga una aplicabilidad que genere desarrollo.

De manera preocupante esta meta no es simple de lograr y frecuentemente escuchamos diversos enfoques y metodologías de cómo revertir las adversidades sobre el aprendizaje de las ciencias.

En este trabajo se ha planteado la forma en la que la implementación de la tecnología por medio de un proyecto integral funge como un recurso valioso para el proceso de enseñanza y aprendizaje, sin embargo, su utilización requiere por parte del profesorado del análisis de un conjunto de factores de diversa índole y el conocimiento de dichos factores le permitirá adaptarlos a su entorno en el que desarrolla su actividad docente que es fundamental en el logro de objetivos exitosos.

Como se ha podido observar, el uso e implementación de tecnología y objetos digitales de aprendizaje integrados en plataformas educativas para el proceso de enseñanza y aprendizaje es en efecto muy importante y de beneficio no solo como elemento de apoyo sino como componente central en el proceso ya que se puede adaptar para usar dichas tecnologías como recursos de investigación, de retroalimentación o bien como componente central del proceso. 
Es importante mencionar que, como todos los demás recursos didácticos existentes, se presentan áreas de oportunidad entre las cuales están aquellas que surgieron de las encuestas aplicadas al finalizar los cursos.

Dentro de las áreas de mejora expuestas por los alumnos, mencionan la importancia de organizar mejor su tiempo para poder destinarlo a las actividades académicas de plataforma, también la infraestructura tecnológica tuvo un papel importante ya que argumentaron que el acceso a una red de datos confiable no siempre les permitía realizar óptimamente sus trabajos.

Con esto se coteja lo que inicialmente se había mencionado que la cantidad, calidad y dosificación de los recursos tecnológicos a usar tienen una influencia preponderante en los resultados finales en la implementación de proyectos educativos usando las tecnologías de la información. 


\section{BIBLIOGRAFÍA}

1. Angulo J, Vales J, Aportes y reflexiones sobre la educación mediada por tecnologías, Instituto de Formación Docente del Estado de Sonora, Tabook Servicios Editoriales e Integrales, S.A. de C.V. (2015).

2. Da Costa M, Integration of Digital Technologies in mathematics Teacher Educación, in Mathematics and Technology, 2017.

3. Díaz B. Angel, TIC en el trabajo del aula, Impacto en la planeación didáctica, Revista Iberoamericana de Educación Superior, Volumen 4, 2013.

4. Fiallo J, Rodriguez G, Situaciones A-didácticas para la enseñanza de la derivada como razón de cambio mediante el uso de software de Geometría Dinámica, Acta Latinoamericana de Matemática Educativa, Volumen 32, 2019.

5. Jimenez S, Tecnología educativa: campos de formación y perfil diferencial, Revista Iberoamericana de Educación Superior, Volumen 5, 2014.

6. Lázaro A. Niurys, Utilización y producción de videotutoriales en matemática, Acta latinoamericana de matemática educativa, Volumen 33, 2020.

7. León Y. Ana, Estrategias de aprendizaje en educación superior en un modelo curricular por competencias, Revista Iberoamericana de Educación Superior, 2015.

8. Pozas D, Alvez M, Experiencia de evaluación con geogebra en un curso de álgebra y geometría para ingeniería, Acta Latinoamericana de Matemática Educativa, Volumen 33, 2020.

9. Padilla E. Gestión y mediación de la asignatura Cálculo Integral en una educación a distancia, Acta Latinoamericana de Matemática Educativa, Volumen 32, 2019

10. Rubio S, León C, Matemática Educativa en la era digital: Recursos educativos Abiertos Integrando Practicas y Tecnologías Digitales, Acta Latinoamericana de Matemática Educativa, Volumen 32, 2019.

11. Santos, M. Potencial didáctico del software dinámico en el aprendizaje de las matemáticas. En Avance y Perspectiva V. 20. Pp. 15-19. Cinvestav. México. 